\title{
Homocysteine-induced proliferation of vascular smooth muscle cells occurs via PTEN hypermethylation and is mitigated by Resveratrol
}

\author{
SHENG-CHAO MA ${ }^{1 *}$, HUI-PING ZHANG ${ }^{2 *}$, YUN JIAO ${ }^{3}$, YAN-HUA WANG ${ }^{1}$, \\ HUI ZHANG ${ }^{1}$, XIAO-LING YANG ${ }^{1}$, AN-NING YANG ${ }^{1}$ and YI-DENG JIANG ${ }^{1}$ \\ ${ }^{1}$ Department of Basic Medicine, Ningxia Medical University; Departments of ${ }^{2}$ Prenatal Diagnosis Center and \\ ${ }^{3}$ Infectious Disease, General Hospital of Ningxia Medical University, Yinchuan, Ningxia 750004, P.R. China
}

Received April 13, 2017; Accepted September 28, 2017

DOI: $10.3892 / \mathrm{mmr} .2018 .8471$

\begin{abstract}
Vascular smooth muscle cell (VSMC) proliferation is a primary pathological event in the development of atherosclerosis (AS), and the presence of homocysteine (Hcy) acts as an independent risk factor for AS. However, the underlying mechanisms remain to be elucidated. Phosphatase and tensin homologue on chromosome 10 (PTEN), is endogenously expressed in VSMCs and induces multiple signaling networks involved in cell proliferation, survival and inflammation, however, the specific role of PTEN is still unknown. The present study detected the proliferation ratio of VSMCs following treatment with Hcy and Resveratrol (RSV). In the $100 \mu \mathrm{M}$ Hcy group, the proliferation ratio increased, and treatment with RSV decreased the proliferation ratio induced by Hcy. Reverse transcription-quantitative polymerase chain reaction and western blotting were used to analyze PTEN expression, RSV treatment was associated with decreased PTEN expression levels in VSMCs. PTEN levels were decreased in Hcy treated cells, and the proliferation ratio of VSMCs were increased following treated with Hcy. To study the mechanism of regulation of PTEN by Hcy, the present study detected PTEN methylation levels in VSMCs, and PTEN DNA methylation levels were demonstrated to be increased in the $100 \mu \mathrm{M}$ Hcy group, whereas treatment with RSV decreased the methylation status. DNA methyltransferase 1 is important role in the regulation of PTEN methylation. Overall, Hcy impacts the methylation status of PTEN, which is involved
\end{abstract}

Correspondence to: Dr Yi-Deng Jiang, Department of Basic Medicine, Ningxia Medical University, 1160 Shengli Street, Yinchuan, Ningxia 750004, P.R. China

E-mail: jydcan@126.com

${ }^{*}$ Contributed equally

Key words: homocysteine, phosphatase and tensin homologue on chromosome 10, vascular smooth muscle cells, Resveratrol, DNA methylation in cell proliferation, and induces the proliferation of VSMCs. This effect is alleviated by treatment with RSV, which exhibits an antagonistic mechanism against Hcy.

\section{Introduction}

Epidemiologic and case control studies have consistently indicated that moderate and mild elevation of plasma homocysteine (Hcy), an intermediate metabolite of methionine, is an independent risk factor in the development of atherosclerosis (AS) (1). Previous studies have intensively focused on the involvement of Hcy in the dysfunction and injury of vascular cells, including vascular smooth muscle cells (VSMCs) $(2,3)$. The authors previously demonstrated that Hcy induces VSMC proliferation, however the underlying mechanisms remain to be elucidated (4).

Hcy is a non-protein, sulfur containing amino acid, which is a metabolic intermediate of the methionine cycle. One of the precursors is $\mathrm{S}$-adenosylmethionine (SAM), the unique methyl group donor for DNA methylation (5). Following the transfer of the methyl group, SAM is transformed into S-adenosylhomocysteine (SAH), which is hydrolyzed to form Hcy (6). DNA methylation refers to the addition of a methyl group to the 5 position of cytosine in the context of a $\mathrm{CpG}$ dinucleotide. Increasing evidence indicates that human diseases, including AS, are either caused or impacted by abnormal methylation (7). The authors previously demonstrated that abnormal DNA methylation of genes including peroxisome proliferator activated receptor- $\alpha$, apolipoprotein E and genomic DNA contribute to the development of AS induced by Hcy $(8,9)$. Phosphatase and tensin homologue on chromosome 10 (PTEN), is a dual-specificity protein and lipid phosphatase that suppresses multiple signaling networks involved in cell proliferation, survival and inflammation (10). PTEN, specifically expressed in VSMCs, is additionally involved in regulation of a variety of physiological and pathological processes, including cell proliferation, differentiation, apoptosis, adhesion and migration (11). PTEN is expressed endogenously in VSMCs, and overexpression of PTEN significantly inhibits both basal and platelet derived growth factor-mediated VSMC proliferation 
and migration (12). PTEN overexpression in VSMCs using adenovirus transfection results in inhibition of cell proliferation and migration induced by angiotensin II (13). However, the direct mechanisms responsible for the PTEN influence on Hcy-induced VSMC proliferation have not been explored.

Resveratrol (RSV), naturally occurring in various plant foods, and particularly in grapes and red wine, is the most investigated and well-known member of this class of compounds (14). It exhibits a protective role against cardiovascular diseases, and is involved in the 'French paradox', characterized by the low incidence of cardiovascular diseases in the French population, despite a high intake of saturated fats, due to their moderate red wine consumption (15). RSV is an effective therapeutic candidate, due to its protective action in vascular walls towards oxidation, inflammation, platelet oxidation and thrombus formation (16). However, the association between Hcy and RSV on PTEN regulation in VSMC proliferation remains to be elucidated.

Therefore, the present study sought to address: i) The role of PTEN DNA methylation in VSMC proliferation induced by Hcy; ii) whether the mechanism of methylation is regulated by DNA methyltransferase (DNMT)1.

\section{Materials and methods}

Cell culture. The T/G HA-VSM cell line was used, which was obtained from American Type Culture Collection (Manassas, VA, USA). Cells were cultured in Dulbecco's modified Eagle's medium-Han's F12 media (DMEM-F12; Gibco; Thermo Fisher Scientific, Inc., Waltham, MA, USA) supplemented with $10 \% \mathrm{FCS}$ at $37^{\circ} \mathrm{C}$ in an incubator with an environment containing $5 \% \mathrm{CO}_{2}$. Hcy was applied at concentrations of $50,100,200$ and $500 \mu \mathrm{M}$ in addition to $30 \mu \mathrm{M}$ folate and vitamin B12 (Sigma-Aldrich; Merck KGaA, Darmstadt, Germany) for $72 \mathrm{~h}$.

Bromodeoxyuridine (BrdU) assay. VSMCs at $5 \times 10^{3}$ cells per well were cultured in triplicate in 96-well plates in 0, 50, 100, 200 and $500 \mu \mathrm{M}$ concentrations of Hcy and antagonist $(30 \mu \mathrm{M}$ folate and $30 \mu \mathrm{M}$ vitamin B12 were added to $100 \mu \mathrm{M}$ Hcy) then incubated for $72 \mathrm{~h}$. Cell proliferation was measured by BrdU incorporation assay using a commercial ELISA kit according to the manufacturer's protocol (cat. no. 11647229001; Roche Diagnostics GmbH, Mannheim, Germany).

Cell viability assessment. MTT (Sigma-Aldrich; Merck $\mathrm{KGaA}$ ) was used for the evaluation of cell viability. The cells were grown in 96-well microtiter plates at a density of $1 \times 10^{4}$ cells in $200 \mu \mathrm{l}$ per well. Subsequently, cells were treated with treated with a range of $10-100 \mu \mathrm{M}$ RSV to determine an optimal concentration. Following incubation for $10 \mathrm{~min}$, the plates were read on a microplate reader (Bio-Rad Laboratories, Inc., Hercules, CA, USA) at $490 \mathrm{~nm}$.

Reverse transcription-quantitative polymerase chain reaction (RT-qPCR) of DNMT1 and PTEN. RNA was then reverse transcribed by using the Revert Aid first strand cDNA synthesis kit (Applied Biosystems; Thermo Fisher Scientific, Inc.); the SYBR-Green kit (Fermentas; Thermo Fisher Scientific, Inc., Pittsburgh, PA, USA) was then used for RT-qPCR analysis.
The primer sequences of the DNMT1 and PTEN genes were as follows: Forward, 5'-AGCTCTTACCTTACCATC-3' and reverse, 5'-CCATCTGATACTCTGAA-3' for DNMT1; and forward, 5'-CAGCATCTTATCCGAGTG-3' and reverse, 5'-GATGGTGGTACAGTCAGA-3' for PTEN. GAPDH was applied as an internal control: Forward, 5'-AGAAGGCTG GGGCTCATTT-3' and reverse, 5'-AGGGGCCACAGTCTT CG-3'. The thermal cycler (Funglyn Biotech, Inc., Toronto, ON, Canada) conditions comprised an initial activation step at $95^{\circ} \mathrm{C}$ for $5 \mathrm{~min}$, followed by a 2 -step PCR program of $95^{\circ} \mathrm{C}$ for $15 \mathrm{sec}$, annealing temperatures for $15 \mathrm{sec}$ and at $72^{\circ} \mathrm{C}$ for $30 \mathrm{sec}$ for 30 cycles. Subsequently, the relative alterations in the mRNA expression levels of DNMT1 and PTEN were determined by fold-change analysis, in which the degree of change was calculated as $2^{-\Delta \Delta \mathrm{Cq}}$, where $\mathrm{Cq}=\left(\mathrm{Cq}_{\mathrm{gene}}-\mathrm{Cq}_{\mathrm{GAPDH}}\right)$ treatment- $\left(\mathrm{Cq}_{\text {gene }}-\mathrm{Cq}_{\mathrm{GAPDH}}\right)$ control (17).

Western blotting for DNMT1 and PTEN. Total proteins were isolated from the cells using cell lysis buffer (Keygen Biotech Co., Ltd., Nanjing, China), then protein concentration was determined via a bicinchoninic acid protein assay kit (KeyGen Biotech, Nanjing, China). Equal amounts of protein $(\sim 80 \mu \mathrm{g})$ and known molecular weight marker were separated by $12 \%$ SDS-PAGE and were transferred to polyvinylidene fluoride membrane by electrophoresis at $300 \mathrm{~mA}$ for $50 \mathrm{~min}$ at $4^{\circ} \mathrm{C}$, the membrane was then blocked in $10 \mathrm{ml} 5 \%$ skimmed milk for $2 \mathrm{~h}$ at room temperature with gentle agitation on a platform shaker. The DNMT1 and PTEN proteins were detected using DNMT1 (cat. no. sc-271729) and PTEN (cat. no. sc-65604) antibodies were obtained from Santa Cruz Biotechnology, Inc, (Dallas, TX, US) diluted 1:500, and $\beta$-actin protein was detected using a rabbit anti-human $\beta$-actin antibody (cat. no. sc-70319, Santa Cruz Biotechnology, Inc.) diluted 1:2,000; all primary antibodies were incubated at $4^{\circ} \mathrm{C}$. The secondary antibody (goat anti-mouse immunoglobulin G-horseradish peroxidase, cat. no. sc-2031, 1:2,000; Santa Cruz Biotechnology, Inc.) was added for $2 \mathrm{~h}$ at room temperature. The protein bands were visualized and analyzed by the Gel Documentation and Analysis System ChemiDoc XRS system with Image Lab software, version 4.1 (Bio-Rad Laboratories, Inc.) and calculated by the gray value of the bands.

Nested methylation-specific (nMS)-PCR for PTEN methylation assay. DNA denaturation and bisulfite conversion processes were integrated into one step by using the EZ DNA Methylation-Gold ${ }^{\mathrm{TM}}$ kit (Zymo Research Corp., Irvine, CA, USA). nMS-PCR consists of two-step PCR amplifications following a standard sodium bisulfite DNA modification. The first step uses an outer primer pair set that does not contain any CpGs. The second-step PCR was conducted with the conventional PCR primers. The primers of the nMS-PCR assays were as follows: i) PTEN-outer primers: Forward, 5'-GTTTTGGTTTGAAGGATAGTAGT-3' and reverse, 5'-AAAAACCCTAAAACTTAATAAAAAC-3'; ii) PTEN-methylated primers: Forward, 5'-TTAGTTTTAGTT AGGATGGTTTCGA-3' and reverse, 5'-GAAAAATAAACC GAAATCCCG-3'; iii) PTEN-unmethylated primers: Forward, 5'-ATTAGTTTTAGTAGGATGGTTTTGA-3' and reverse, 5'-CAA AAA ATAAACCAAAATCCCAC-3'. DNA bands were visualized by ultraviolet illumination, and the percentage 
A

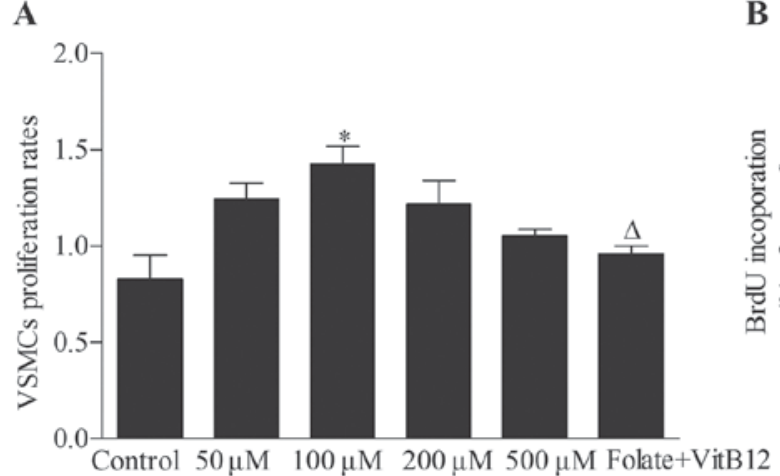

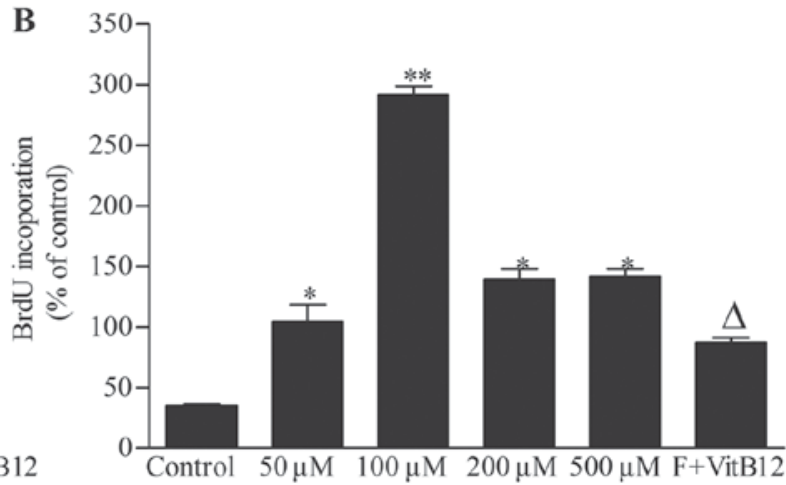

Figure 1. Hcy increases the proliferation ratio of VSMCs. (A) Following treatment with different concentrations of Hcy, MTT was used to detect the proliferation of VSMCs. (B) VSMC proliferation was analyzed using a BrdU assay, following cell pre-treatment with various concentrations of Hcy. Folate and vitamin B12 group: Cells were treated with $100 \mu \mathrm{M}$ Hcy, $30 \mu \mathrm{M}$ folate and $30 \mu \mathrm{M}$ vitamin $\mathrm{B} 12 .{ }^{*} \mathrm{P}<0.05,{ }^{* *} \mathrm{P}<0.01$ vs. control; ${ }^{\Delta} \mathrm{P}<0.05$ vs. $100 \mu \mathrm{M}$ Hcy group. Hcy, homocysteine; VSMC, vascular smooth muscle cells; BrdU, bromodeoxyuridine.

of methylation was calculated by using the following formula: Methylation $\%=$ methylation/(methylation+unmethylation) x $100 \%$.

Cell transfection. The recombinant plasmids EGFP-N1-PTEN/DNMT1 were established obtain from Hanbio Biotechnology Co., Ltd., (Shanghai, China). Cells were plated to 70-90\% confluence at the time of transfection; $100 \mathrm{ng}$ plasmid and $0.5 \mu \mathrm{l}$ for DNA-lipid complexes were prepared and incubated for $30 \mathrm{~min}$. The DNA-lipid complexed were then applied to cells. Then, the VSMCs were transfected with 3-5 $\mu \mathrm{g} / \mu 1$ recombinant plasmids: EGFP-N1-PTEN/DNMT1. EGFP-N1-PTEN/DNMT1 molecule-Lipofectamine ${ }^{\circledR} 2000$ (Invitrogen; Thermo Fisher Scientific, Inc.) complexes were established and then added to the plate wells, where the VSMCs had grown to $80-90 \%$ confluence. The transfection efficiency was determined by detecting the fluorescence intensity via an inverted fluorescence phase contrast microscope (Olympus IX71, Olympus Corporation, Tokyo, Japan). The transfected VSMCs were treated with Hcy for $24 \mathrm{~h}$ and mRNA expression of PTEN/DNMT1 was detected with RT-qPCR (17). Western blotting was carried out to examine the protein expression of PTEN/DNMT1 (17).

Statistical analysis. Prism 5.0 (GraphPad Software, Inc., La Jolla, CA, USA) was used for data processing. Data are expressed as the mean \pm standard deviation. Each experiment was repeated three times. Statistical comparison of each parameter between two groups was performed using the paired Student's t-test. One-way analysis of variance was used to compare the means of multiple groups, followed by Dunnett's test. $\mathrm{P}<0.05$ was considered to indicate a statistically significant difference.

\section{Results}

Hcy promotes the proliferation of VSMCs. In order to investigate the effect of Hcy on VSMCs, the present study detected the cell proliferation ratio following cell treatment with Hcy for 72 h, using an MTT assay. As presented in Fig. 1A, the proliferation of VSMCs was increased in the cells which were co-incubated with different concentrations of Hcy, compared with control group, and in $100 \mu \mathrm{M}$ Hcy it was increased 1.72-fold $(\mathrm{P}<0.05)$. The proliferation ratio in the folate and vitamin B12 group treated with $100 \mu \mathrm{M}$ Hcy was decreased by $35.46 \%(\mathrm{P}<0.05)$. Furthermore, the effect of Hcy on VSMC proliferation was evaluated with a BrdU incorporation assay to determine the effects of Hcy on DNA synthesis, and it was demonstrated that $100 \mu \mathrm{M}$ Hcy treatment significantly promoted DNA synthesis (Fig. 1B), whereas in the folate and vitamin $\mathrm{B} 12$ group, DNA synthesis was decreased $(\mathrm{P}<0.05)$. These data indicated that Hcy promoted the proliferation ratio, and this effect was counteracted with folate and vitamin B12.

Hcy decreases PTEN expression in VSMCs. PTEN is ranked the most mutated tumor suppressor gene, which regulates numerous cellular processes, including cell cycle, adhesion, migration, proliferation and cell survival (18). To study whether Hcy regulates PTEN expression, and if this was responsible for the VSMC proliferation, the present study detected PTEN expression levels in VSMCs following treatment with Hcy for $72 \mathrm{~h}$. As presented in Fig. 2, PTEN mRNA was decreased by $27.5,34.7$ and $46.7 \%$ in 100, 200 and $500 \mu \mathrm{M}$ Hcy groups respectively, compared with control group $(\mathrm{P}<0.05, \mathrm{P}<0.01)$. PTEN protein levels in VSMCs decreased by 28.6, 54.3 and $71.4 \%$ in 100,200 and $500 \mu \mathrm{M}$ Hcy groups, compared with control group $(\mathrm{P}<0.05, \mathrm{P}<0.01)$, whereas in folate and vitamin B12 group, PTEN protein levels were increased 1.44-fold, compared with $100 \mu \mathrm{M}$ Hcy group $(\mathrm{P}<0.05)$. Therefore, PTEN may have an important role in VSMCs proliferation induced by Hcy.

RSV antagonizes proliferation of VSMCs induced by Hcy. RSV has biological activities, and similar to estrogen, is known to have several effects on cardiovascular disease and malignancies (19). The present study co-incubated VSMCs with different concentrations of RSV and $100 \mu \mathrm{M}$ Hcy for $72 \mathrm{~h}$ at $37^{\circ} \mathrm{C}$, then detected the VSMC proliferation ratio by MTT, as presented in Fig. 3. Compared with $100 \mu \mathrm{M}$ Hcy group, the proliferation ratio of VSMCs was decreased by $45.16,53.76$ and $63.44 \%$ in 20, 50 and $100 \mu \mathrm{M}$ RSV groups $(\mathrm{P}<0.05, \mathrm{P}<0.01)$, respectively. Compared with $100 \mu \mathrm{M}$ RSV group, the proliferation ratio in the folate and vitamin B12 group was increased by 1.62-fold 


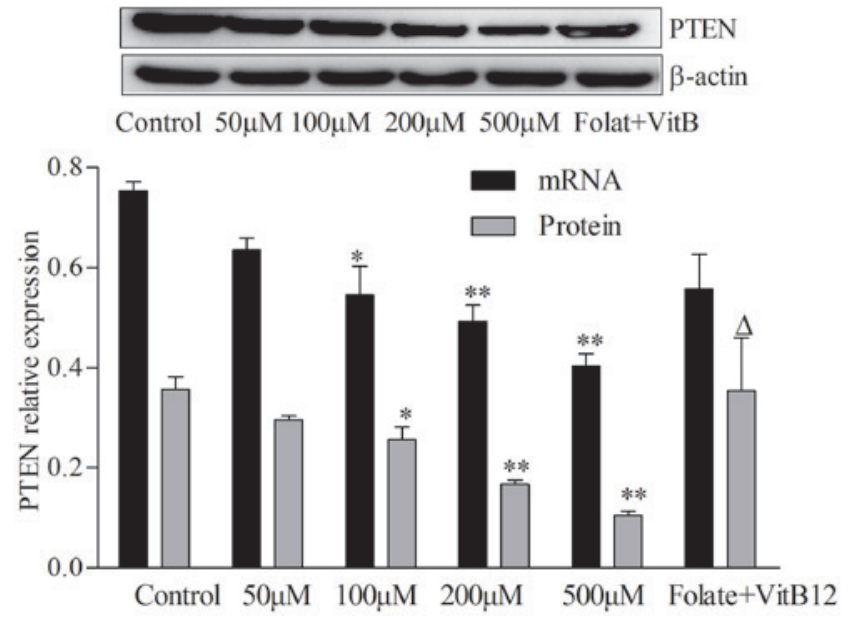

Figure 2. PTEN expression is downregulated in VSMCs treated with Hcy. Following incubation with different concentrations of Hcy for $72 \mathrm{~h}$, the PTEN mRNA level was detected by reverse transcription-quantitative polymerase chain reaction, and the expression of PTEN protein analyzed by western blotting. Folate and vitamin B12 group: Cells were treated with $100 \mu \mathrm{M} \mathrm{Hcy}$, $30 \mu \mathrm{M}$ folate and $30 \mu \mathrm{M}$ vitamin $\mathrm{B} 12 .{ }^{*} \mathrm{P}<0.05,{ }^{* *} \mathrm{P}<0.01$ vs. control; ${ }^{\Delta} \mathrm{P}<0.05$ vs. $100 \mu \mathrm{M}$ Hcy groups. Hcy, homocysteine; VSMC, vascular smooth muscle cells; PTEN, phosphatase and tensin homologue on chromosome 10.

$(\mathrm{P}<0.05)$. Notably, compared with $100 \mu \mathrm{M}$ Hcy group, the folate and vitamin B12 group proliferation ratio of VSMCs was decreased by $40.86 \%(\mathrm{P}<0.05)$. These data indicated that RSV acted as an antagonist which decreased the proliferation ratio of VSMCs, which was previously increased when induced by Hcy. The inhibitory effect of RSV was more potent compared with folate and vitamin B12.

Methylation status of PTEN in VSMCs. DNA methylation is important in the development of AS induced by Hcy (20). To investigate whether PTEN DNA methylation alterations occur in VSMC proliferation induced by Hcy, the present study detected PTEN DNA methylation by nMS-PCR, following cell treatment with Hcy and various antagonistic compounds for $72 \mathrm{~h}$. As presented in Fig. 4A, compared with control group, PTEN DNA methylation levels were increased 8.56-fold in $100 \mu \mathrm{M}$ Hcy group $(\mathrm{P}<0.01)$, and 2.56-fold in folate and vitamin $\mathrm{B} 12$ group $(\mathrm{P}<0.05)$. Compared with $100 \mu \mathrm{M}$ Hcy group, PTEN DNA methylation levels decreased by 79.2 and $70.1 \%$ in RSV $(\mathrm{P}<0.01)$ and folate and vitamin $\mathrm{B} 12$ groups $(\mathrm{P}<0.01)$, respectively. These data suggested that PTEN DNA methylation was affected by Hcy in VSMCs and this may act as the primary reason for proliferation of VSMCs induced by Hcy.

DNMT1 catalyzes the transfer of methyl groups to DNA from SAM. In mammals, DNMT1 is the maintenance methyltransferase, which preferentially methylates unmethylated or hemi-methylated double-stranded DNA. To further study the regulation mechanism of PTEN DNA methylation, the expression of DNMT1 in VSMCs was analyzed by RT-qPCR and western blotting. As presented in Fig. 4B, DNMT1 mRNA levels were detected in VSMCs. Compared with control group, DNMT1 mRNA increased 3.28-fold in $100 \mu \mathrm{M}$ Hcy group $(\mathrm{P}<0.01)$, whereas compared with $100 \mu \mathrm{M}$ Hcy group, DNMT1 mRNA levels decreased 47.4 and $57.9 \%$ in RSV $(\mathrm{P}<0.01)$ and folate and vitamin B12 groups $(\mathrm{P}<0.01)$, respectively. DNMT1

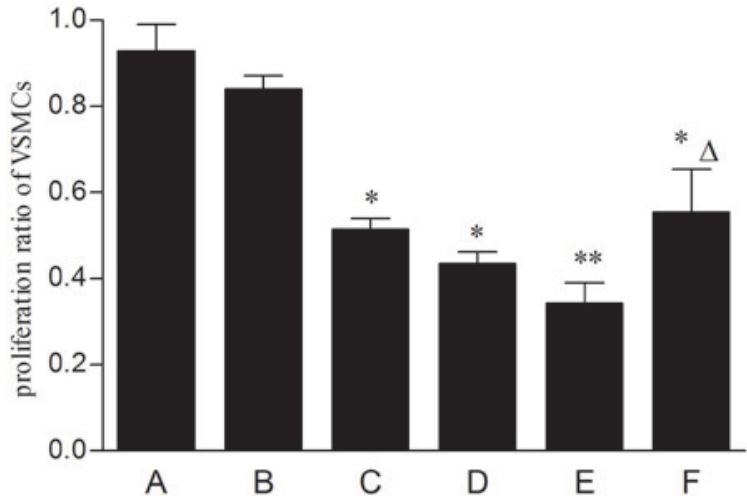

Figure 3. Resveratrol antagonistic effects on proliferation of VSMCs induced by Hcy. VSMCs were treated with Hcy and different concentrations of Resveratrol for $72 \mathrm{~h}$, then the proliferation ratio of VSMCs was detected by MTT. A, $100 \mu \mathrm{M}$ Hcy group, as control group; B, $100 \mu \mathrm{M}$ Hcy $+10 \mu \mathrm{M}$ Resveratrol; C, $100 \mu \mathrm{M}$ Hcy $+20 \mu \mathrm{M}$ Resveratrol; D, $100 \mu \mathrm{M}$ Hcy+50 $\mu \mathrm{M}$ Resveratrol; E, $100 \mu \mathrm{M}$ Hcy $+100 \mu \mathrm{M}$ Resveratrol; F, cells were treated with $100 \mu \mathrm{M}$ Hcy, $30 \mu \mathrm{M}$ folate and $30 \mu \mathrm{M}$ vitamin $\mathrm{B} 12 .{ }^{*} \mathrm{P}<0.05,{ }^{* *} \mathrm{P}<0.01$, compared with $100 \mu \mathrm{M}$ Hcy group; ${ }^{\Delta} \mathrm{P}<0.05$ vs. $100 \mu \mathrm{M}$ Hcy $+100 \mu \mathrm{M}$ Resveratrol group. Hcy, homocysteine; VSMC, vascular smooth muscle cells.

protein expression in VSMCs exhibited the same trends as the DNMT1 mRNA levels. Compared with control group, DNMT1 protein expression increased 2.81-fold in the100 $\mu \mathrm{M}$ Hcy group $(\mathrm{P}<0.01)$, whereas compared with $100 \mu \mathrm{M}$ Hcy group, DNMT1 protein levels decreased by 47.4 and $43.4 \%$ in RSV $(\mathrm{P}<0.01)$ and folate and vitamin $\mathrm{B} 12$ groups $(\mathrm{P}<0.01)$, respectively.

To identify whether DNMT1 was a key molecule in regulation of PTEN DNA methylation in VSMC proliferation induced by Hcy, the levels of DNMT1 were overexpressed. The recombination vector of DNMT1 was transfected into untreated VSMCs, and the observed green fluorescence verified that the recombined vector had been successfully transfected into VSMCs (Fig. 4C). The expression levels of DNMT1 were detected with RT-qPCR and western blotting. As presented in Fig. 4D, DNMT1 mRNA and protein expression levels were increased in the overexpression group by 3.57-fold and 2.03-fold ( $\mathrm{P}<0.01)$, compared with control group, and these data indicated that the transfection of the overexpression recombination vector DNMT1 had been successful. Following this, PTEN DNA methylation was analyzed with nMS-PCR. As presented in Fig. 4E, compared with control group, PTEN DNA methylation was increased 2-fold $(\mathrm{P}<0.05)$ in DNMT1 overexpression group and 2.59-fold $(\mathrm{P}<0.01)$ in DNMT1 overexpression and Hcy group. Overall, these data suggested that DNMT1 has an important role in regulating PTEN DNA methylation in VSMCs, induced by Hcy.

PTEN exhibits a primary role in the proliferation of VSMCs induced by Hcy. To further verify the role of PTEN in VSMC proliferation resulting from Hcy, a recombination PTEN overexpression vector was transfected into untreated VSMCs and proliferation of VSMCs induced by Hcy was analyzed, as presented in Fig. 5. Following transfection with recombination vector of PTEN, green fluorescence in VSMCs was observed, and the levels of PTEN mRNA and protein were increased 1.91-fold $(\mathrm{P}<0.01)$ and 2.27 -fold $(\mathrm{P}<0.01)$ in the PTEN recombination group (Fig. $5 \mathrm{~A}$ and $\mathrm{B}$ ). 

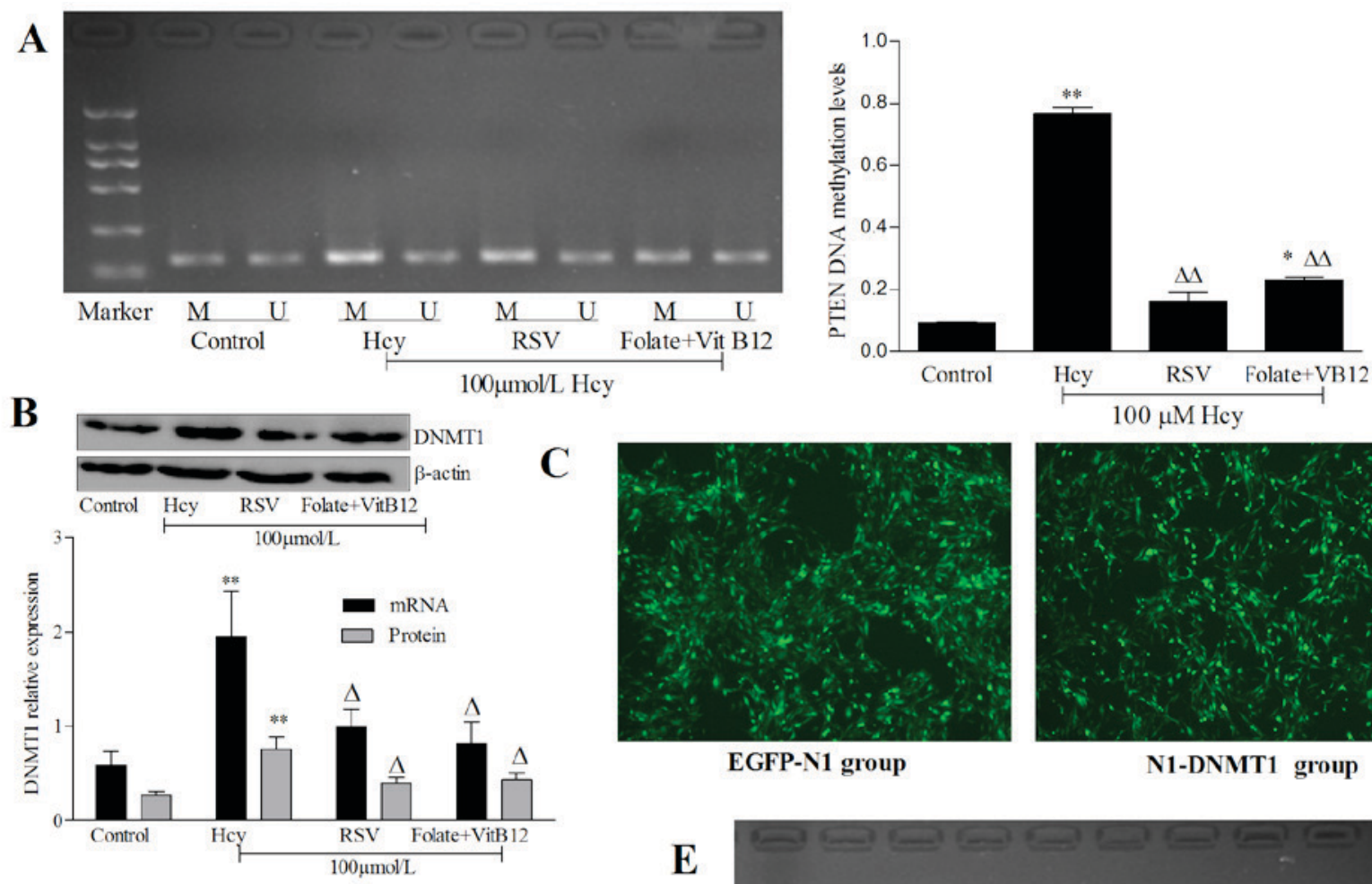

C

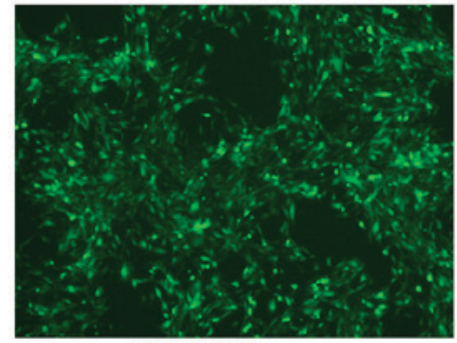

EGFP-N1 group

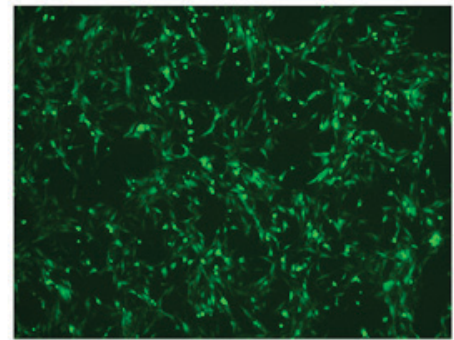

N1-DNMT1 group

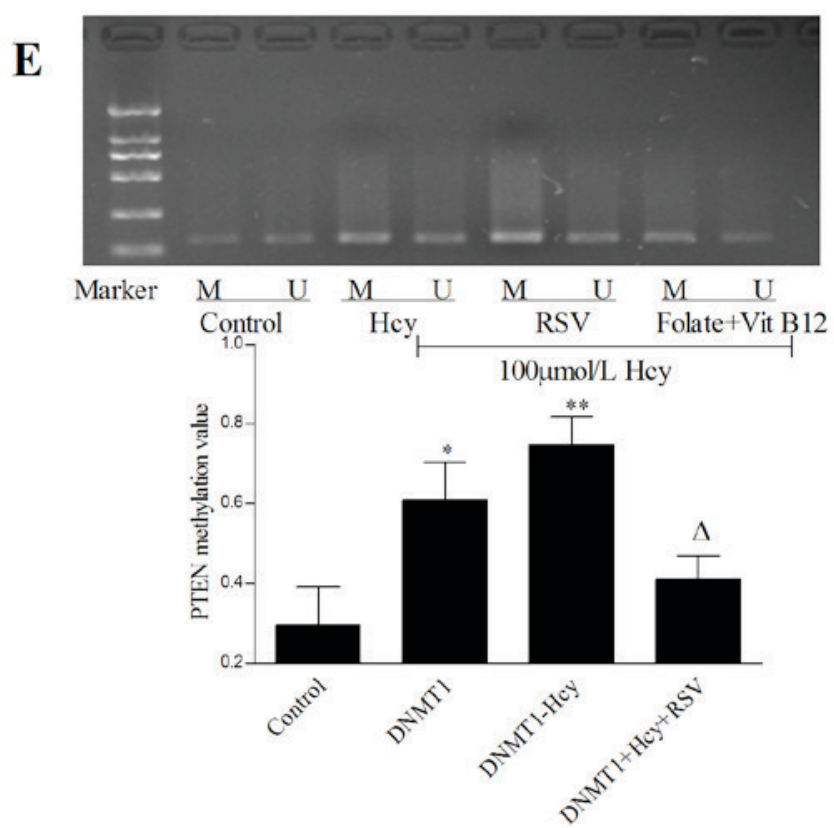

Figure 4. PTEN DNA methylation and its regulation mechanism in VSMCs induced by Hcy. (A) PTEN DNA methylation levels in VSMCs detected by nested methylation-specific PCR, following cell treatment with Hcy and other antagonists for $72 \mathrm{~h}$. (B) DNMT1 expression levels in VSMCs detected by RT-qPCR and western blotting, following cell treatment with Hcy and other antagonists for $72 \mathrm{~h}$. (C) pEGFP-N1-DNMT1 was transfected into VSMCs using Lipofectamine $^{\circledR} 2000$, and detected with fluorescence microscopy (magnification, x1,000). (D) DNMT1 mRNA and protein levels in VSMCs analyzed by RT-qPCR and western blotting, following cell transfection with DNMT1 overexpression vector. (E) Levels of PTEN DNA methylation in VSMCs, following cell transfection with recombination vector of DNMT1, then co-incubation with Hcy for 72 h. Control group, untreated cells; Hcy group: Cells treated with $100 \mu \mathrm{M}$ Hcy for $72 \mathrm{~h}$; RSV group, cells treated with $100 \mu \mathrm{M}$ Hcy and $100 \mu \mathrm{M}$ Resveratrol for $72 \mathrm{~h}$; Folate and vitamin B12 group, cells treated with $100 \mu \mathrm{M}$ Hcy, $30 \mu \mathrm{M}$ folate and $30 \mu \mathrm{M}$ vitamin B12; EGFP-N1, pEGFP-N1 control vector; DNMT1-N1, recombination vector of DNMT1; DNMT1 group, cells transfected with recombination vector of DNMT1; DNMT1+Hcy group, cells transfected with recombination vector of DNMT1, then treated with $100 \mu \mathrm{M}$ Hcy for $72 \mathrm{~h}$; DNMT1+Hcy+RSV group, cells transfected with recombination vector of DNMT1, then treated with $100 \mu \mathrm{M}$ Hcy and $100 \mu \mathrm{M}$ Resveratrol for $72 \mathrm{~h}$; ${ }^{*} \mathrm{P}<0.05,{ }^{* *} \mathrm{P}<0.01$, vs. control group; ${ }^{\wedge} \mathrm{P}<0.05,{ }^{\Delta \Delta} \mathrm{P}<0.01$ vs. $100 \mu \mathrm{M}$ Hcy group; ${ }^{*} \mathrm{P}<0.05$ vs. pEGFP-N1 vector group. M, methylation; U, unmethylated; Marker, DNA marker (top to bottom, 2,000, 1,000, 750, 500, 250 and 100 bp). Hcy, homocysteine; VSMC, vascular smooth muscle cells; PTEN, phosphatase and tensin homologue on chromosome 10; DNMT1, DNA methyltransferase; RSV, Resveratrol; RT-qPCR, reverse transcription-quantitative polymerase chain reaction.

Following this, the proliferation ratio of VSMCs was detected following cell transfection with the PTEN recombination vector. As presented in Fig. $5 \mathrm{C}$, the proliferation ratio was increased 4.75-fold $(\mathrm{P}<0.01), 3.25$-fold $(\mathrm{P}<0.01)$ and 2.1 -fold $(\mathrm{P}<0.01)$ in Hcy, PTEN overexpression cells treated with Hcy and PTEN overexpression cells treated with Hcy and RSV groups, respectively. Compared with $100 \mu \mathrm{M}$ Hcy group, in PTEN overexpression cells treated with Hcy and PTEN overexpression cells treated with Hcy and RSV, the proliferation ratio was decreased by $33.3 \%(\mathrm{P}<0.05)$ and $56.6 \%(\mathrm{P}<0.05)$. 
A

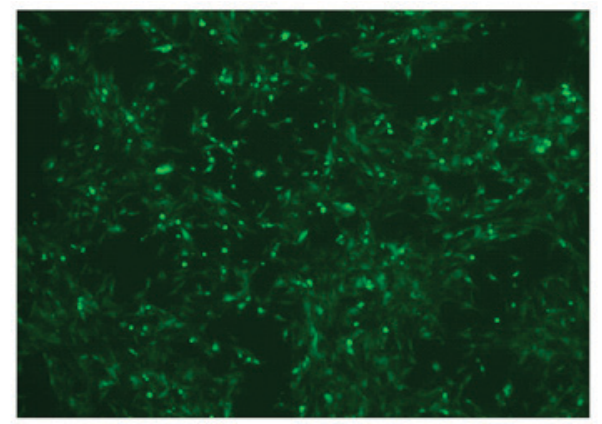

EGFP-N1 group

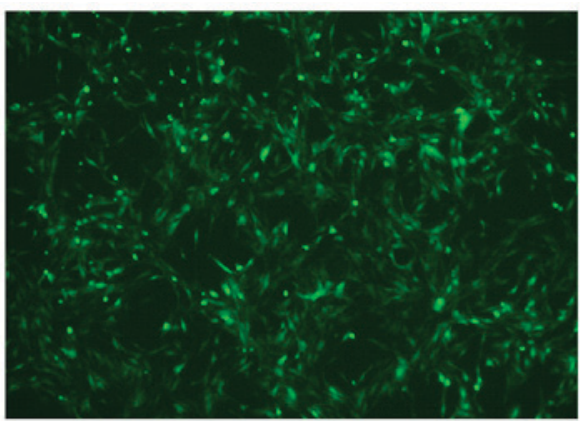

N1-PTEN group

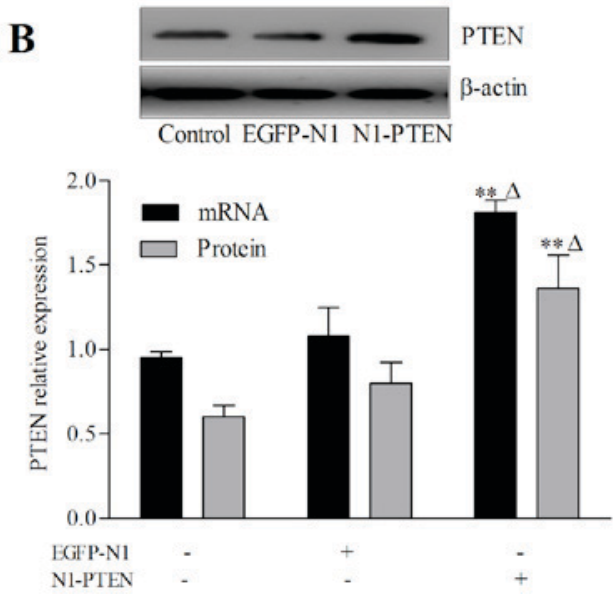

C

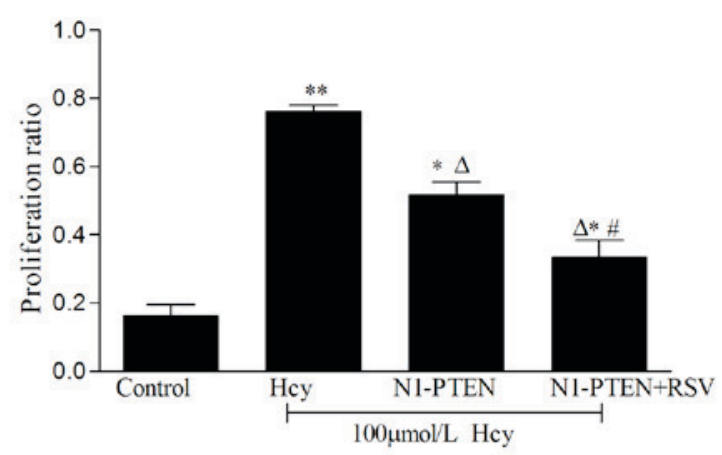

Figure 5. PTEN is a pivotal molecule in proliferation of VSMCs induced by Hcy. (A) pEGFP-N1 and pEGFP-N1-PTEN were transfected into VSMC using Lipofectamine ${ }^{\mathrm{TM}}$ 2000, and detected with fluorescence microscopy (magnification, x100). (B) Expression levels of PTEN in VSMCs, following cell transfection with recombination vector. mRNA and protein levels were analyzed with reverse transcription-quantitative polymerase chain reaction and western blotting. (C) Proliferation ratio of VSMCs was detected by MTT, following cell transfection with recombination vector PTEN, and then co-incubation with Hcy and other antagonistic compounds. EGFP-N1, pEGFP-N1 control vector; PTEN-N1, recombination vector of PTEN; Hcy group, cells treated with $100 \mu$ M Hcy for $72 \mathrm{~h}$; N1-PTEN+Hcy group, cells transfected with recombination vector of PTEN, then treated with $100 \mu \mathrm{M}$ Hcy for $72 \mathrm{~h}$; N-PTEN+Hcy+RSV group, cells transfected with recombination vector of PTEN, then treated with $100 \mu \mathrm{M}$ Hcy and $100 \mu \mathrm{M}$ Resveratrol for $72 \mathrm{~h}$; ${ }^{*} \mathrm{P}<0.05,{ }^{* *} \mathrm{P}<0.01 \mathrm{vs}$. control group; ${ }^{\Delta} \mathrm{P}<0.05$ vs. pEGFP-N1 vector group; ${ }^{~} \mathrm{P}<0.05$, vs. $100 \mu \mathrm{M}$ Hcy group. Hcy, homocysteine; VSMC, vascular smooth muscle cells; PTEN, phosphatase and tensin homologue on chromosome 10 .

These results suggested that PTEN is important in proliferation of VSMCs which is induced by Hcy, and RSV may relieve this nocuous effect of Hcy.

\section{Discussion}

It has previously been demonstrated that elevated Hcy levels are a diagnosed cause of VSMC proliferation and further promote the formation of AS (21). The present study demonstrated that Hcy induced the proliferation of VSMCs, which may contribute to development of AS. Clinical studies regarding treatment with RSV are not as promising as the preclinical findings, with regards to the beneficial effects of RSV on AS $(22,23)$. The present study demonstrated that Hcy promoted VSMC proliferation, and decreased PTEN expression via an increase in PTEN DNA methylation levels. DNMT1 was important in this process, and RSV relieved the pathological process via a promotion of PTEN expression levels.

As an important independent risk factor of AS, Hcy stimulates VSMC proliferation, which has been demonstrated in previous studies $(24,25)$. To investigate the effect of Hcy on VSMCs, the present study detected the cell proliferation ratio following cell treatment with Hcy for
$72 \mathrm{~h}$ using an MTT assay, and the proliferation of VSMCs was observed to be increased with different concentrations of Hcy. PTEN is a tumor suppressor gene, which regulates various cellular processes, including cell cycle, adhesion, migration, proliferation and cell survival (26). PTEN has recently been demonstrated to have an important role in cells of the cardiovascular system (27). Schwartzbauer and Robbins (28) demonstrated that PTEN regulates cardiac myocyte hypertrophy and survival. The present study demonstrated that PTEN expression levels were decreased in Hcy groups, at the mRNA and protein level.

RSV is a naturally occurring polyphenolic phytoalexin derived from plants such as Polygonum cuspidatum and grapes, it has anti-aging, -apoptotic, -tumorigenic, -oxidant, and -inflammatory properties (29). Guo (30) reported that RSV inhibits high glucose-induced oxidative stress and VSMC proliferation by suppressing reactive oxygen species generation, nicotinamide adenine dinucleotide phosphate oxidase, AKT Serine/Threonine Kinase phosphorylation, p38 mitogen activated protein kinase/c-Jun $\mathrm{N}$-terminal kinase/extracellular signal-regulated kinase phosphorylation, and IкB- $\alpha$ and nuclear factor- $11 \kappa \mathrm{B}$ activities, however the effects of RSV in anti-proliferation of VSMCs still remain unclear. The present 
study co-incubated VSMCs with different concentrations of RSV and $100 \mu \mathrm{M}$ Hcy for $72 \mathrm{~h}$, and demonstrated that the VSMC proliferation ratio was decreased in different concentrations of RSV groups. These data indicated that RSV acted as an antagonist which decreased the proliferation ratio of VSMCs induced by Hcy.

DNA methylation serves as an important mechanism that controls gene expression in AS (31). As previously demonstrated, PTEN DNA methylation levels are significantly decreased in Hcy groups. In Hcy metabolism, SAM is a metabolic intermediate which is synthesized from methionine catalysed by methionine adenosyltransferase, providing methyl group moieties in several dozens of transmethylation reactions (32). SAM is converted into SAH that is the sole metabolic precursor of Hcy in a reversible reaction catalyzed by SAH hydrolase, and inhibits DNMT1 (33). The present study demonstrated that PTEN DNA methylation levels were increased in $100 \mu \mathrm{M}$ Hcy group and decreased in RSV group. The role of DNMT1 in the regulation of PTEN was also observed. DNMT1, 3a, 3b have all previously been detected, however only DNMT1 exhibits an important role in this process (34). To test whether DNMT1 had an effect on PTEN promoter hypermethylation, overexpression of DNMT1 and PTEN occurred. Following transfection with pDNMT1, the methylation levels of PTEN significantly increased.

In conclusion, Hcy impacted the methylation status of PTEN involved in cell proliferation, and induced the proliferation of VSMCs, whereas RSV alleviated VSMC proliferation by antagonism of Hcy. The data from the present study provided evidence for the mechanisms of VSMC proliferation in AS induced by Hcy, and may act as a potential diagnostic marker for AS induced by Hcy.

\section{Acknowledgements}

The present study was supported by the National Natural Science Foundation of China (grant nos. 81570452, 81560084, 81670416, 81660088 and 81760076).

\section{References}

1. Devasia AJ, Joy B and Tarey SD: Serum homocysteine as a risk factor for carotid intimal thickening in acute stroke: A cross sectional observational study. Ann Indian Acad Neurol 19: 48-51, 2016.

2. Liu T, Lin J, Ju T, Chu L and Zhang L: Vascular smooth muscle cell differentiation to an osteogenic phenotype involves matrix metalloproteinase-2 modulation by homocysteine. Mol Cell Biochem 406: 139-149, 2015.

3. Liu X, Shen J, Zhan R, Wang X, Wang X, Zhang Z, Leng X, Yang Z and Qian L: Proteomic analysis of homocysteine induced proliferation of cultured neonatal rat vascular smooth muscle cells. Biochim Biophys Acta 1794 $177-184,2009$.

4. Han XB, Zhang HP, Cao CJ, Wang YH, Tian J, Yang XL, Yang AN, Wang J, Jiang YD and Xu H: Aberrant DNA methylation of the PDGF gene in homocysteine-mediated VSMC proliferation and its underlying mechanism. Mol Med Rep 10: 947-954, 2014

5. Taysi S, Keles MS, Gumustekin K, Akyuz M, Boyuk A, Cikman $\mathrm{O}$ and Bakan N: Plasma homocysteine and liver tissue $\mathrm{S}$-adenosylmethionine, $\mathrm{S}$-adenosylhomocysteine status in vitamin B6-deficient rats. Eur Rev Med Pharmacol Sci 19: 154-160, 2015.
6. Elshorbagy AK, Jernerén F, Samocha-Bonet D, Refsum H and Heilbronn LK: Serum S-adenosylmethionine, but not methionine, increases in response to overfeeding in humans. Nutr Diabetes 6: e192, 2016.

7. Wang L, Fu X, Peng X, Xiao Z, Li Z, Chen G and Wang X: DNA methylation profiling reveals correlation of differential methylation patterns with gene expression in human epilepsy. J Mol Neurosci 59: 68-77, 2016.

8. Yang AN, Zhang HP, Sun Y, Yang XL, Wang N, Zhu G, Zhang H, $\mathrm{Xu} \mathrm{H}$, Ma SC, Zhang Y, et al: High-methionine diets accelerate atherosclerosis by HHcy-mediated FABP4 gene demethylation pathway via DNMT1 in ApoE(-/-) mice. FEBS Lett 589: 3998-4009, 2015.

9. Yideng J, Zhihong L, Jiantuan X, Jun C, Guizhong L and Shuren W: Homocysteine-mediated PPARalpha,gamma DNA methylation and its potential pathogenic mechanism in monocytes. DNA Cell Biol 27: 143-150, 2008.

10. Chen WJ, Lin KH, Lai YJ, Yang SH and Pang JH: Protective effect of propylthiouracil independent of its hypothyroid effect on atherogenesis in cholesterol-fed rabbits: PTEN induction and inhibition of vascular smooth muscle cell proliferation and migration. Circulation 110: 1313-1319, 2004.

11. Sedding DG, Widmer-Teske R, Mueller A, Stieger P, Daniel JM, Gündüz D, Pullamsetti S, Nef H, Moellmann H, Troidl C, et al: Role of the phosphatase PTEN in early vascular remodeling. PLoS One 8: e55445, 2013.

12. Rice KM, Uddemarri S, Desai DH, Morrison RG, Harris R, Wright GL and Blough ER: PGF2alpha-associated vascular smooth muscle hypertrophy is ROS dependent and involves the activation of mTOR, p70S6k, and PTEN. Prostaglandins Other Lipid Mediat 85: 49-57, 2008.

13. Hu C, Liu S, Sun Y, Shi G and Li Y: Effect of recombinant hPTEN gene expression on PDGF induced VSMC proliferation. Cell Biochem Biophys 70: 1185-1190, 2014.

14. Moradi H and Vaziri ND: Effect of Resveratrol on progression of polycystic kidney disease: A case of cautious optimism. Nephrol Dial Transplant 31: 1755-1758, 2016.

15. Pieszka M, Szczurek P, Ropka-Molik K, Oczkowicz M and Pieszka M: The role of Resveratrol in the regulation of cell metabolism-a review. Postepy Hig Med Dosw (Online) 70: 117-123, 2016 (In Polish).

16. Fišar Z, Hroudová J, Singh N, Kopřivová A and Macečková D: Effect of simvastatin, coenzyme Q10, Resveratrol, acetylcysteine and acetylcarnitine on mitochondrial respiration. Folia Biol (Praha) 62: 53-66, 2016.

17. Ma SC, Cao JC, Zhang HP, Jiao Y, Zhang H, He YY, Wang YH, Yang XL, Yang AN, Tian J, et al: Aberrant promoter methylation of multiple genes in VSMC proliferation induced by Hcy. Mol Med Rep: Sep 19, 2017 (Epub ahead of print).

18. Hu C, Liu S, Sun Y, Shi G and Li Y: Effect of recombinant hPTEN gene expression on PDGF induced VSMC proliferation. Cell Biochem Biophys 70: 1185-1190, 2014.

19. Dong $X$, Yu LG, Sun R, Cheng YN, Cao H, Yang KM, Dong YN, Wu Y and Guo XL: Inhibition of PTEN expression and activity by angiotensin II induces proliferation and migration of vascular smooth muscle cells. J Cell Biochem 114: 174-182, 2013.

20. Greißel A, Culmes M, Napieralski R, Wagner E, Gebhard H, Schmitt M, Zimmermann A, Eckstein HH, Zernecke A and Pelisek J: Alternation of histone and DNA methylation in human atherosclerotic carotid plaques. Thromb Haemost 114: 390-402, 2015.

21. Meng L, Liu L, Zhou C, Pan S, Zhai X, Jiang C, Guo Y, Ji Z, Chi J, Peng F and Guo H: Polyphenols and polypeptides in Chinese rice wine inhibit homocysteine-induced proliferation and migration of vascular smooth muscle cells. J Cardiovasc Pharmacol 67: 482-490, 2016.

22. Swiader A, Nahapetyan H, Faccini J, D'Angelo R, Mucher E, Elbaz M, Boya P and Vindis C: Mitophagy acts as a safeguard mechanism against human vascular smooth muscle cell apoptosis induced by atherogenic lipids. Oncotarget 7: 28821-28835, 2016.

23. Chen WJ, Pang JH, Lin KH, Lee DY, Hsu LA and Kuo CT: Propylthiouracil, independent of its antithyroid effect, promotes vascular smooth muscle cells differentiation via PTEN induction. Basic Res Cardiol 105: 19-28, 2010.

24. Chawla V, Simionescu A, Langan EM III and LaBerge M: Influence of clinically relevant mechanical forces on vascular smooth muscle cells under chronic high glucose: An in vitro dynamic disease model. Ann Vasc Surg 34: 212-226, 2016. 
25. Jiang C, Zhang H, Zhang W, Kong W, Zhu Y, Zhang H, Xu Q, Li Y and Wang X: Homocysteine promotes vascular smooth muscle cell migration by induction of the adipokine resistin. Am J Physiol Cell Physiol 297: C1466-C1476, 2009.

26. Bonnevaux $H$, Lemaitre $O$, Vincent L, Levit MN, Windenberger F Halley F, Delorme C, Lengauer C, Garcia-Echeverria C and Virone-Oddos A: Concomitant inhibition of PI3K $\beta$ and BRAF or MEK in PTEN-deficient/BRAF-mutant melanoma treatment: Preclinical assessment of SAR260301 oral PI3K $\beta$-selective inhibitor. Mol Cancer Ther 15: 1460-1471, 2016.

27. Morais CL, Guedes LB, Hicks J, Baras AS, De Marzo AM and Lotan TL: ERG and PTEN status of isolated high-grade PIN occurring in cystoprostatectomy specimens without invasive prostatic adenocarcinoma. Hum Pathol 55: 117-125, 2016.

28. Schwartzbauer G and Robbins J: The tumor suppressor gene PTEN can regulate cardiac hypertrophy and survival. J Biol Chem 276: 35786-35793, 2001.

29. Tang F, Guo S, Liao H, Yu P, Wang L, Song X, Chen J and Yang Q: Resveratrol enhances neurite outgrowth and synaptogenesis via sonic hedgehog signaling following oxygen-glucose deprivation/reoxygenation injury. Cell Physiol Biochem 43 : 852-869, 2017.
30. Guo R, Li W, Liu B, Li S, Zhang B and Xu Y: Resveratrol protects vascular smooth muscle cells against high glucose-induced oxidative stress and cell proliferation in vitro. Med Sci Monit Basic Res 20: 82-92, 2014.

31. Ma SC, Zhang HP, Kong FQ, Zhang H, Yang C, He YY, Wang YH, Yang AN, Tian J, Yang XL, et al: Integration of gene expression and DNA methylation profiles provides a molecular subtype for risk assessment in atherosclerosis. Mol Med Rep 13: 4791-4799, 2016

32. Hai Z and Zuo W: Aberrant DNA methylation in the pathogenesis of atherosclerosis. Clin Chim Acta 456: 69-74, 2016.

33. Grimaldi V, Vietri MT, Schiano C, Picascia A, De Pascale MR, Fiorito C, Casamassimi A and Napoli C: Epigenetic reprogramming in atherosclerosis. Curr Atheroscler Rep 17: 476, 2015.

34. Benetatos L and Vartholomatos G: On the potential role of DNMT1 in acute myeloid leukemia and myelodysplastic syndromes: Not another mutated epigenetic driver. Ann Hematol 95: 1571-1582, 2016. 